Dialectologia. Special issue, IX (2021), 1-5.

ISSN: 2013-2247

\title{
NUEVAS APROXIMACIONES
}

\section{AL ATLAS LINGÜÍSTICO DE LA PENÍNSULA IBÉRICA (ALPI) ${ }^{1}$}

\author{
Maria-Pilar PEREA \\ Universitat de Barcelona \\ mpilar.perea@ub.edu
}

\section{Resumen}

He aquí una breve presentación de los artículos publicados en este número extraordinario dedicado al Atlas Lingüístico de la Península Ibérica $(A L P I)$ y de los resultados obtenidos a partir de la explotación de sus datos mediante la adopción de perspectivas y enfoques diferentes. Los trabajos se centran grosso modo en cuatro temáticas generales: a) el estudio de elementos intrínsecos al $A L P I$; b) la descripción del léxico; c) la paremiología y la fraseología; d) el análisis monográfico de aspectos relacionados con la descripción dialectal, los gentilicios, la morfología verbal y la fonética.

\section{NEW APPROACHES TO THE ATLAS LINGÜÍSTICO DE LA PENÍNSULA IBÉRICA (ALPI)}

\section{Abstract}

We briefly introduce the papers in this special issue of Dialectologia devoted to dialect the Atlas Lingüístico de la Península Ibérica $(A L P I)$ and to the results obtained from different perspectives. The papers focus roughly on four general subjects: a) the study of intrinsic elements to $A L P I$; $b$ ) the description of the lexicon; c) paremiology and phraseology; d) the monographic analysis of aspects related to dialect description, demonyms, verbal morphology and phonetics.

Este es el segundo número extraordinario ${ }^{2}$ que la revista electrónica Dialectologia dedica al Atlas Lingüístico de la Península Ibérica (ALPI).

\footnotetext{
${ }^{1}$ Este número extraordinario se adscribe al proyecto coordinado PGC2018-095077-B-C41/C42/C43/C44, El Atlas Lingüístico de la Península Ibérica: edición digital y análisis de datos, financiado por el Ministerio de Ciencia, Innovación y Universidades.

${ }^{2}$ Véase Linguistic Atlas of the Iberian Peninsula (ALPI): Progress and Perspectives, Dialectologia, special issue, III, 2012 <http://www.publicacions.ub.edu/revistes/dialectologiaSP2012/>.
} 
Es de sobras conocido que, después de su publicación parcial, en 1962, el ALPI vivió en una etapa de relativo silencio hasta que, a finales de los años noventa, este atlas de amplio espectro, concebido por Ramón Menéndez Pidal, publicado por Tomás Navarro Tomás, y que contó con la colaboración como encuestadores de Aurelio M. Espinosa (hijo), Lorenzo Castellano, Francesc de B. Moll, Manuel Sanchis Guarner, Aníbal Otero, Armando Nobre de Gusmão y Luis F. Lindley Cintra, empezó a hacerse oír de nuevo gracias al tesón del profesor de la Universidad de Western Ontario, David Heap. Él supo contagiar su entusiasmo a otros investigadores y desde entonces, a través de distintos proyectos sucesivos, un equipo de base formado por este profesor, junto con Pilar García Mouton (coord.), Inés Fernández-Ordóñez, Maria-Pilar Perea, João Saramago y Xulio Sousa, se ha propuesto la publicación de los numerosos materiales inéditos en Internet con la finalidad de dar a conocer a los estudiosos y al público en general la riqueza inherente a este atlas.

Se trataba, y este es el objetivo general de nuestro actual proyecto, de la edición digital rigurosa de los materiales del $A L P I$, sin excluir, naturalmente, la posibilidad de publicar una selección de términos representativamente relevantes y su correspondiente cartografía en un volumen en papel. La tarea de entrada de datos y su revisión se ha dilatado en el tiempo, cosa perfectamente justificable debido a la ingente cantidad de información encerrada en más de mil de cuadernos que recopilan información lingüística de 528 localidades. Durante el proceso de introducción y revisión de los materiales se han ido descubriendo los tesoros que esconde el $A L P I$ y este número extraordinario pretende dar a conocer algunos de ellos.

Es muy frecuente asociar de manera inmediata los atlas con dos ámbitos lingüísticos: el léxico y la fonética. Sin embargo, lo cierto es que el $A L P I$ incorpora muchísima más información, no solo de tipo sintáctico, a partir de las frases que fueron elicitadas a los informadores, sino también morfológico, antropológico o relacionado con la cultura popular.

Gracias a su alcance, que abraza todo el ámbito peninsular, excepto el País Vasco y el valle de Arán, pueden hacerse estudios de carácter general pero también de carácter parcelario, que se basan en las áreas que configuran las diversas lenguas (y sus correspondientes dialectos) habladas en la península. 
Dialectologia. Special issue, IX (2021), 1-5.

ISSN: 2013-2247

Desusados y olvidados algunos vocablos que el $A L P I$ registra, este atlas es, sin embargo, un testimonio documental inmejorable del habla de un determinado periodo temporal, la cual, sometida a la acción inexorable del cambio lingüístico no deja de modificarse. Las formas constituyen a su vez un excelente punto de partida para efectuar comparaciones con soluciones actuales o con registros más antiguos que se hallen documentados en trabajos anteriores. A su vez, además de la comparación diacrónica, y debido a que incluye soluciones de todo el territorio citado, constituye también una herramienta óptima para contrastar sus resultados con atlas o diccionarios de otras áreas lingüísticas. Si uno de los fines de los mapas dialectales es registrar la distribución de las formas recogidas en los puntos encuestados de un determinado territorio, otro y mucho más importante es efectuar una interpretación adecuada de los resultados. Y esto es lo que pretenden los artículos que se presentan a continuación.

\section{Los artículos}

Los artículos que se publican en este número extraordinario son una muestra de la heterogeneidad de los materiales y de las posibilidades de estudio y de comparación que ofrece el Atlas Lingüístico de la Península Ibérica.

La secuencia en que se presentan responde grosso modo a un criterio de ordenación temática: a) elementos intrínsecos al $A L P I ; b)$ el léxico; c) la paremiología y la fraseología; d) aspectos monográficos relacionados con la descripción dialectal, los gentilicios, la morfología verbal y la fonética.

Con relación al análisis de elementos intrínsecos al $A L P I$, Eloi Bellés en "Perfiles sociolingüísticos de los informantes de los atlas dialectales catalanes" analiza y compara, desde un punto de vista sociolingüístico, las características de los sujetos que se interrogaron en las encuestas lingüísticas para la elaboración de los tres principales atlas dialectales catalanes: el Atlas lingüístic de Catalunya $(A L C)$, el Atlas lingüístico de la Península Ibérica $(A L P I)$ y el Atles lingüístic del domini català $(A L D C)$; con relación a los estudios dialectales del gallego, Francisco Dubert-García muestra, en "Lo que el 
ALPI le ofrecía a la lingüística gallega en 1962", la información relevante que aportó el primer volumen del $A L P I$ con relación al gallego popular y presenta algunas hipótesis que pretenden explicar por qué esta obra no fue, en su momento, tomada en consideración.

Con relación al léxico, y en especial respecto al estudio de determinados campos semánticos, "De usureros, cornudos y glotones en el léxico catalán recogido por el $A L P I^{\prime \prime}$, de Joan Fontana i Tous, estudia estas las denominaciones correspondientes al usurero (núm. 729), al cornudo (núm. 731) y al glotón (núm. 734) en las 111 localidades que configuran el dominio lingüístico catalán y el área castellana de tierras valencianas; las soluciones que se indican constatan el incalculable valor histórico y lexicográfico del ALPI. El artículo de José Enrique Gargallo Gil, "Nombres de vientos en catalán continental: creatividad léxica y territorio", constituye un elenco de nombres de vientos del ámbito continental del catalán que se caracterizan por ofrecer referencias específicas al territorio. La inclusión de refranes relacionados con la meteorología popular enlaza con el campo temático siguiente: la paremiología.

Pilar García Mouton, con el artículo "El arco iris en el Atlas Lingüístico de la Península Ibérica", inicia una serie de tres textos relacionados con proverbios y locuciones; en su caso, pone de manifiesto el conglomerado de refranes que se encuentran en las páginas de los cuadernos del $A L P I$, y se centra, en particular, en el estudio del arco iris. Diversos aspectos asociados con la fraseología, en comparación con otros corpus y repertorios lexicográficos, son desarrollados en los trabajos "La fraseología con verbos de movimiento en el ALPI: acerca de llevar a cuestas, de Jacinto González Cobas, y "La fraseología en el ALPI: el caso de andar a gatas y sus variantes", de Ana Serradilla Castaño.

El apartado de monografías se abre con "La aportación de Ibiza al Atlas Lingüístico de la Península Ibérica (ALPI)", de Antonio Torres, que ilustra detalladamente $\mathrm{y}$ desde un punto de vista contrastivo las numerosas formas específicas documentadas sobre el dialecto ibicenco. Igualmente des de la perspectiva comparativa, Maria-Pilar Perea estudia la estructura de los sufijos que forman los gentilicios catalanes, junto con su distribución y uso, a partir de los datos que aportan los cuadernos de campo de Antoni M. Alcover, el Diccionari català-valencià-balear y el 
Atlas Lingüístico de la Península Ibérica. Des del ámbito de la morfología verbal, y en este caso a través del contraste entre el Atlas Lingüístico de la Península Ibérica y el Atlas Lingüístico Galego, Clara Lago Caamaño y Xulio Sousa, en "Cambio lingüístico y variación en el gallego rural del siglo XX: los perfectos verbales", describen los resultados de los cambios lingüísticos acaecidos en el gallego rural del siglo pasado, comparando la información existente en estos dos atlas. Finalmente, en "Evolución y distribución de /s/ en coda silábica en español en el Atlas Lingüístico de la Península Ibérica", de Patricia Ribas Marí, se describe la glotalización y pérdida de /s/ en posición implosiva en el español peninsular desde una perspectiva geolingüística, mostrando la extensión geográfica de este fenómeno y sus diferentes etapas evolutivas.

Confiemos que, con motivo de su difusión y del futuro interés que suscite, la revista Dialectologia pueda dedicar al Atlas Lingüístico de la Península Ibérica dentro de unos años un tercer número extraordinario. 